\title{
Effects on the Ventilation of a Two-Storey Building under Different Thermal Conditions
}

\author{
Tim Röder ${ }^{1, *}$, Paul Mathis ${ }^{1}$, and Dirk Müller ${ }^{1}$ \\ ${ }^{1}$ RWTH Aachen University, E.ON Energy Research Center, Institute for Energy Efficient Buildings and Indoor Climate, Germany
}

\begin{abstract}
In this paper it is shown how the air flow rate of decentralized ventilation devices can be affected by a staircase of a two-storey building under different thermal conditions. Since these devices need local fans for supplying the requested volume flow, pressure loads have a significant impact on the delivered volume flow rates. Regarding this, the study comprises two analyses: First, a CFD-model is developed to simulate the ventilation air flow through a simplified staircase. By varying parameters for rooms' temperature and ventilation direction, the hydrostatic pressure in the staircase is evaluated. The simulations - characterized by high Archimedes numbers - are successfully validated with findings from preliminary work. In a second part, the pressure conditions inside the staircase are referred to outside conditions. Consequently, a static pressure difference at the ventilation device on each storey can be observed.

We found that the deliverable volume air flow rate can decrease up to $10 \%$ from the nominal flow rate due to temperature differences between the storeys and outside. Therefore, heat recovery and ventilation effectiveness may also be impaired.
\end{abstract}

\section{Introduction}

Over the last couple of years, decentralized ventilation devices have become very popular for domestic buildings. Since these devices need local fans for supplying the requested volume flow, pressure loads have a significant impact on the delivered volume flow rates and consequently on the air flow distribution in the entire building.

Some of these ventilation systems work in an alternating manner, which means that each ventilation device operates either in a supplying or exhausting mode repeatedly. Thus, two single devices form one complete system. Heat recovery is realized with a heat storage medium within each device, allowing the supply and exhaust air flow being geometrically separated from each other. Consequently, a pair of ventilation devices can be integrated in two different rooms inside a building, even on different floors. For that, an open staircase helps to balance the volume air flows on each floor.

Depending on several geometrical and thermal conditions in the staircase, pressure conditions can occur at the ventilation devices, which affect the deliverable volume flow rate. Ultimately, this issue may decrease heat recovery and ventilation effectiveness.

For these reasons, the work in this paper deals with two central topics to evaluate the resulting pressure differences at the ventilation devices:

The aim of the first study in this paper is to analyse the behaviour of the flow through the staircase inside the building. Therefore, the staircase is further reduced geometrically by isolating it from the building. Now, the flow can be simplified as a vertical flow leading to a more general study. With this approach, CFD-simulations are performed easily under a range of different thermal and geometrical parameter, which are introduced later on.

The second goal is to transfer the findings from the previous study into mathematical correlations. The pressure conditions inside the building are considered in relation to the outside pressure conditions.

As a conclusion, the pressure differences are assessed with regard to their impact on the volume air flow rate at the ventilation devices.

In the literature, there are a few studies, where the flow through a staircase is examined. Unfortunately, they often focus on special geometries under special topics. Some interesting measurements in terms of the mixing process due to buoyancy have been conducted by Peppes et. al. [12]. Because also infiltration through walls affects their results, findings are not useable in this paper.

Since the paper's work concentrates on the flow between two thermal zones, findings can also be related to the Rayleigh-Bénard convection. Several studies in this field with size ratios comparable to a staircase were conducted at the Ilmenauer Fass (Barrel of Ilmenau) by du Puits et. al. [3]. Hence, the experimental setup does not allow an overlay of the convective flow with a vertical flow field, but a detailed examination of the boundary layer flow instead.

A more applicable setup is the one Linke [4] built up. Although Linke's study is performed with heated and cooled walls, in this paper we also expect that flow structures change depending on different thermal conditions and volume flow rates similar to Fig. 1. This is

\footnotetext{
* Corresponding author: troeder@eonerc.rwth-aachen.de
} 
also consistent with Rietschel's results [5]. Wildeboer et. al. [6] did the same investigations with both experiments and CFD analysis. Their observations are also in good agreement. However, the main weakness in their study is that the numerical setup was not able to ensure a vertical flow field as boundary conditions. Therefore, the established stability criteria of the flow field regarding Archimedes and Reynolds number must be questioned.

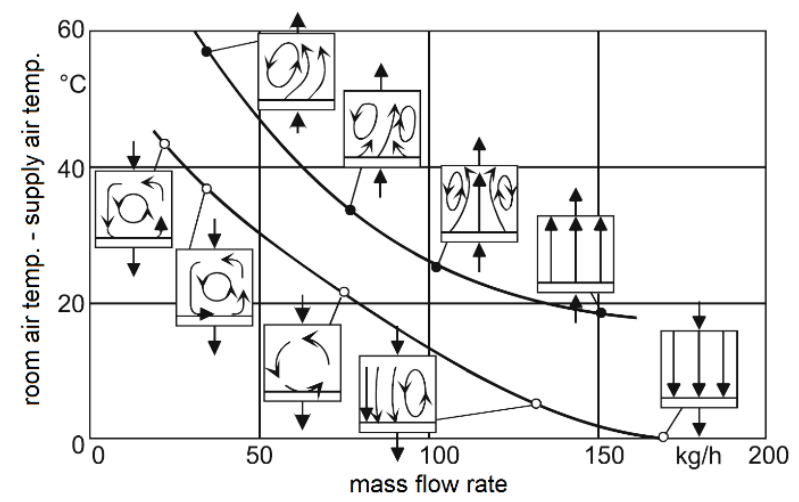

Fig. 1. Flow structures in a vertical flow under thermal conditions [4].

Since Archimedes number is often defined as equation (1), it can be interpreted as the ratio of buoyancy forces to convective forces.

$$
A r=\frac{L \cdot g \cdot \Delta T}{v^{2} \cdot T}
$$

Regarding dimensionless numbers such as Archimedes, the main issue of respecting self-similarity is how to choose well-suited characteristic parameters. However, air flows are usually a three-dimensional problem, a single characteristic length scale is not sufficient to describe the full problem set. [7]

\section{Methods}

For the investigation in this paper, a domestic building is investigated (Fig. 2). In order to keep complexity to a minimum, it is assumed that the building is only equipped with two decentralized ventilation devices, one on each floor.

The schematic way of flow is illustrated in Fig. 2 pointing from the second to the first floor, although the flow direction between the decentralized ventilation devices can be in both directions downstairs and upstairs.

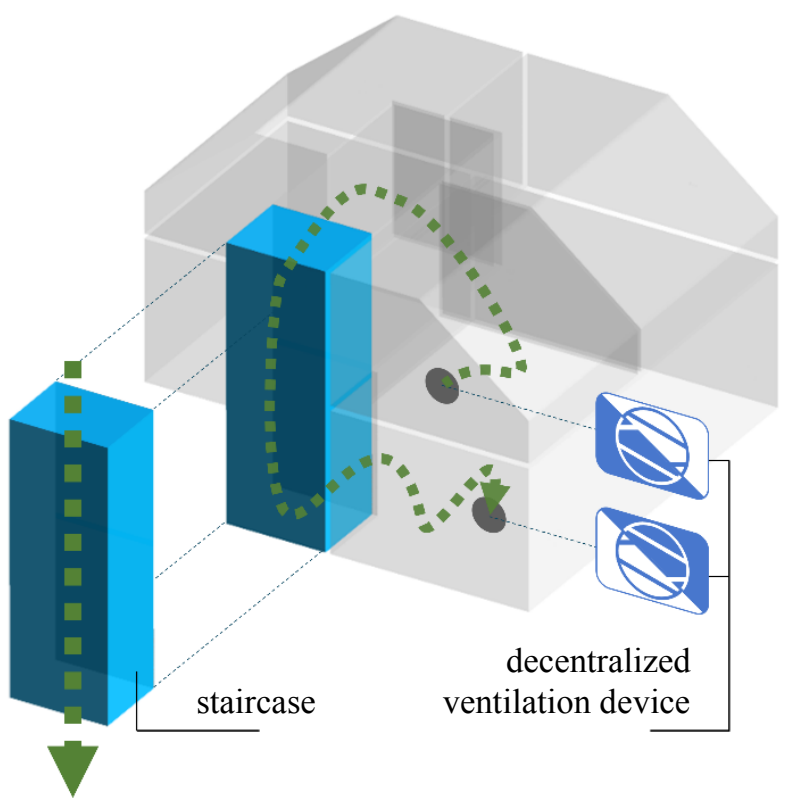

Fig. 2. Staircase (blue) inside of a domestic building with schematic way of flow (green).

\subsection{CFD model of the staircase}

In order to perform simulations of the air flow, the staircase extracted from the building has to be transferred to a CFD environment. The resulting model is shown in Fig. 3. The software application used for simulation is ANSYS ${ }^{\circledR}$ Academic Research CFX, Release 19.2.

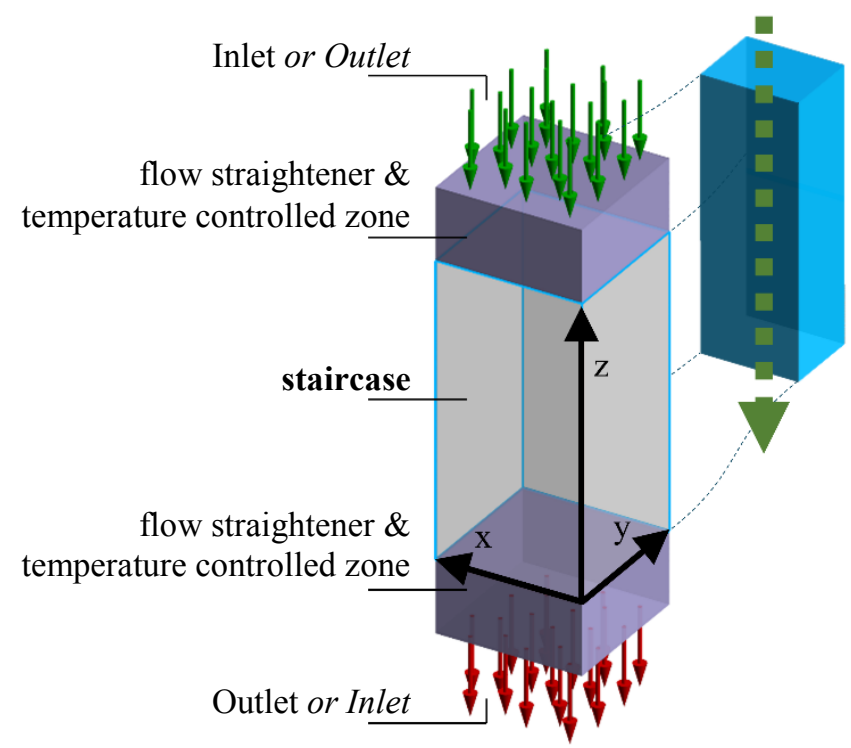

Fig. 3. Simplified staircase model in CFD.

Actually, the entire model is divided into three parts of which the middle one is representing the staircase while the two at the top and the bottom have to fulfil special requirements.

Geometrically, the model is an empty rectangular box. The dimensions of the staircase volume according to the Cartesian coordinate system are length (x), depth (y) and 
height (z), which are all variable parameters. At the top and bottom, the air enters or leaves the domain perpendicular to the boundary. All bounding surfaces have free slip conditions to prevent effects of boundary layers.

Since there is no wall-bounded flow, the study is carried out with the widely used RNG k- $\varepsilon$-RANS model under thermal conditions, which has great advantages with shear-layer flows [8]. The numerical mesh is based on hexahedral finite volumes of $0.1 \mathrm{~m}$ size. In the area where the staircase zone adjoins the two special sections a mesh refinement is conducted to take into account high flow gradients, which can occur there. Hence, the cell size is decreased by factor 4 . Depending on the dimension of the staircase, this leads to a number of cells between 2000 and 5600 .

The sections at the top and at the bottom satisfy two main functions: First, they ensure a constant temperature level on each side of the staircase. Considering this, the behaviour of the flow can be investigated depending on different temperature gradients within the staircase. The control of the temperature is achieved by implementing a heat source formulation, which is introducing the required amount of heat to keep constant the nominal temperature for each mesh cell individually. The second task is due to numerical reasons. CFD-simulations perform best regarding numerical stability, if a velocity inlet and a pressure outlet are applied at once. [9]

Since, the pressure outlet is not able to guarantee a homogeneous, vertical flow distribution like the velocity inlet does, an artificial flow straightener is necessary (Fig. 4 , left). This is realized with a porous medium approach, which is adjusted as follows:

The vertical flow can be achieved by implementing an additional pressure drop for transverse velocity components, which is higher than it is in streamwise direction by a factor of $10^{3}$. Consequentially, by suppressing the transverse velocity components, no pressure compensation is possible in this direction anymore. This leads to a vertical flow but also to a highly inhomogeneous flow field, because the outlet closes partially to prevent a recirculating flow (Fig. 4, middle).

To fix this issue, a small pressure drop in streamwise direction based on the velocity magnitude is also applied. This ensures homogenization as soon as the air enters the porous medium (Fig. 4, right).

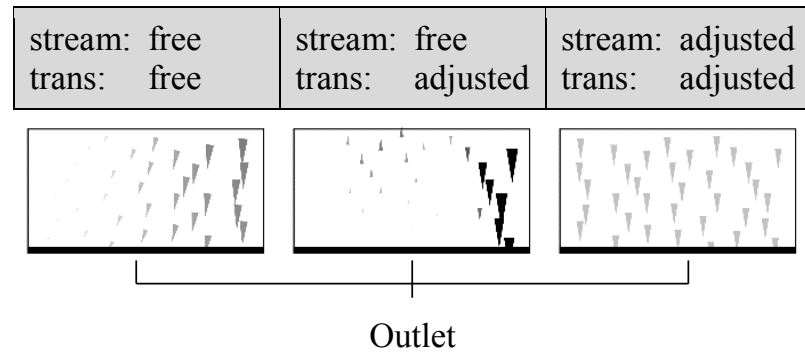

Fig. 4. Flow direction control (streamwise and transverse) near the outlet boundary (sideview).

By avoiding feedback effects on the outlet boundary in the CFD model, this simulation environment is able to fulfil the requirements to investigate a vertical flow field between two different thermal zones.

\subsection{Pressure level}

The second aspect of this paper is how the air flow rate is affected at the ventilation devices. To evaluate which pressure differences at the ventilation devices can occur, Fig. 5 helps to clarify the single pressure levels qualitatively.

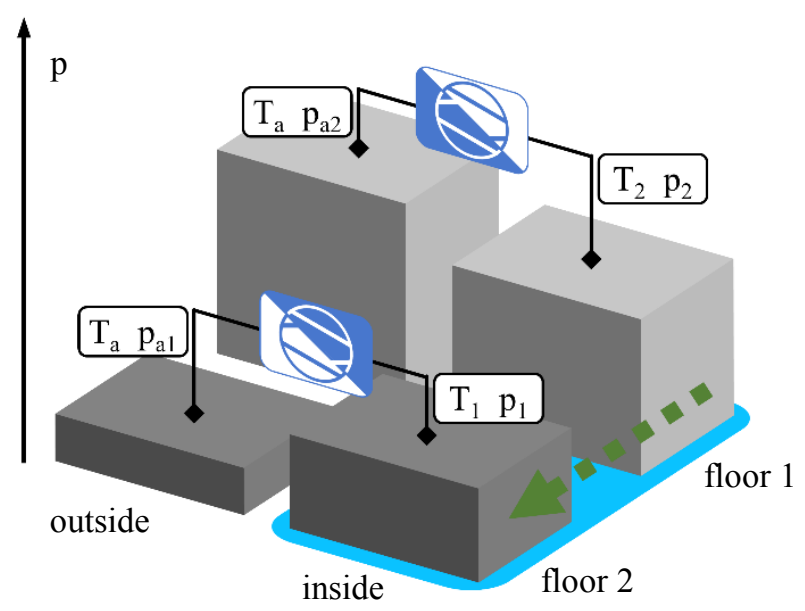

Fig. 5. Pressure levels at ventilation devices

Obviously, the pressure difference between the storeys shown in this example is smaller inside the building than outside $\left(p_{1}-p_{2}<p_{a 1}-p_{a 2}\right)$. Considering the hydrostatic pressure this always occurs, when the mean temperature inside the building is higher than outside. This is typical for wintertime.

However, assuming that both the characteristic fan curve of the ventilation devices is linear and continuity equation must be adhered between the two devices, equation (2) always applies.

$$
p_{1}-p_{a 1}=p_{a 2}-p_{2}=\Delta p
$$

Focussing the ventilation device at the first floor, the pressure difference inhibits the functionality of the device in exhaust mode, so that the air flow rate is decreasing. On the other hand, the device is supported in supply mode due to a negative pressure gradient. The air flow rate is increasing.

As the pressure difference at the ventilation device in the second floor is the opposite, the behaviour is opposed. Therefore, the marked flow direction mentioned in the figures before stands for an operation mode, which leads to reduced volume flow rates in wintertime but to higher ones in summer.

Estimating this impact quantitatively, the hydrostatic pressure outside and inside the building is formulated in equation (3) in general. Gravity $g$ effects vertical downward in negative z-direction. 


$$
\frac{\partial p}{\partial z}=-\rho g
$$

Introducing the ideal gas law into equation (3) leads to equation (4).

$$
\frac{\partial p}{p}=-\frac{g}{R \cdot T(z)} \cdot \partial z
$$

Depending on the temperature profile $T(z)$ outside and inside the building, the hydrostatic pressure can be evaluated as well as $\Delta p$.

\section{Results}

In order of the presented methods, CFD simulations are conducted and evaluated regarding temperature and pressure conditions. To analyse the effects due to buoyancy forces, in our setup the temperature at the bottom area is always higher than at the top.

\subsection{Analysis of the staircase air flow}

Typically, decentralized ventilation devices deliver a volume flow rate up to $80 \mathrm{~m}^{3} / \mathrm{h}$, which corresponds to air velocities less than $10 \mathrm{~mm} / \mathrm{s}$ through the staircase.

Assuming temperature gradients of around $5 \mathrm{~K}$, results from the CFD simulation show a characteristic behaviour, which is the same in nearly all cases. Exemplarily, in Fig. 6 the velocity vectors and isolines of the temperature are plotted as well as the relative velocity to the entry velocity. The flow direction in this case is downwards.
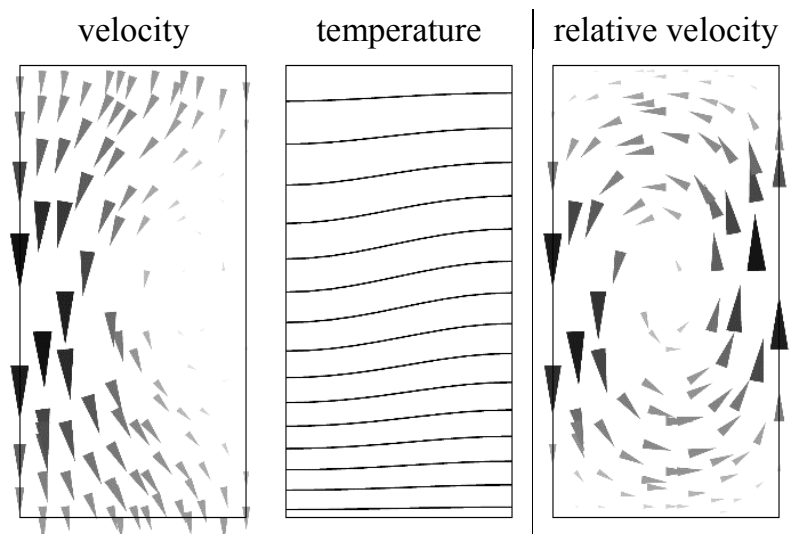

Fig. 6. Plot of velocity and temperature with dominant buoyancy (center plane).

It can be seen that the velocity field is influenced significantly by the buoyancy flow, which forces a vortex system highlighted in the right plot. Accordingly, due to the enhanced mixing effect, a constant gradient over the staircase's height is obtained. This confirms previous findings in the literature presented before.

However, as this mixing process depends on several parameters, the next step is to evaluate under which conditions the vortex system is suppressed. For that, under different geometrical and thermal parameters the volume flow rate is increased until the heat transfer due to convection dominates other effects.

In analogy to the previous case, Fig. 7 represents a volume flow rate, where no relative velocity components appear anymore. Following, there is no gradient in the temperature profile within the staircase anymore.

In the following the velocity magnitude, where the characteristic behaviour switches between the cases under given boundary conditions, is defined as $v_{\text {limit }}$.

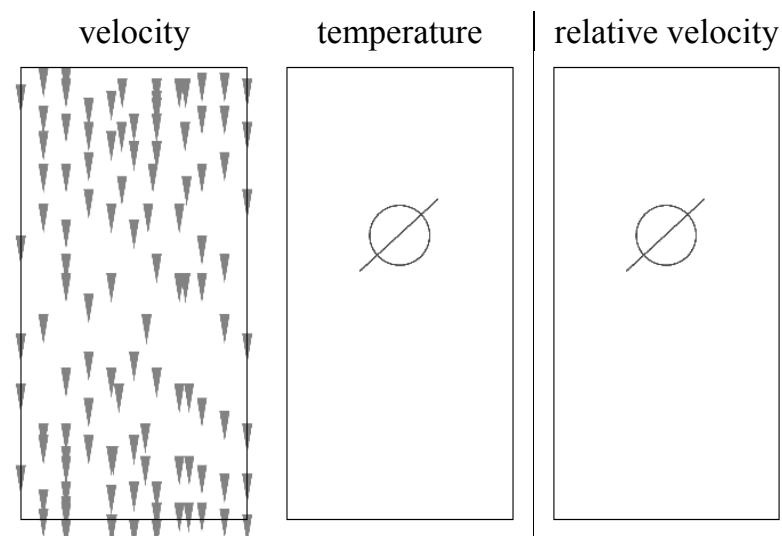

Fig. 7. Plot of velocity and temperature with suppressed buoyancy (center plane).

As it has been found out, there are two types of how the flow develops in the channel. For further investigations, $v_{\text {limit }}$ is evaluated in a case study. For that, flow conditions are changed according to Table 1.

Table 1: Parameter of the case study

\begin{tabular}{|l|c|}
\hline temperature difference & $4 ; 8 ; 12[\mathrm{~K}]$ \\
\hline flow direction & up- $(\uparrow) ;$ downwards $(\downarrow)$ \\
\hline height (h) & $1 ; 1.5 ; 2[\mathrm{~m}]$ \\
\hline depth (d) & $0.4 ; 0.8[\mathrm{~m}]$ \\
\hline
\end{tabular}

The staircase length is fixed to $1 \mathrm{~m}$, the upper temperature is $28^{\circ} \mathrm{C}$ in any case, while the temperature difference is defined as $\Delta T=\left|T_{1}-T_{2}\right|$.

Fig. 8 and Fig. 9 show the limiting velocity magnitude $v_{\text {limit }}$ depending on the given parameters. From the results, there are several findings to point out:

The flow direction shows no remarkable impact on $v_{\text {limit }}$ except in the case of $h=2 \mathrm{~m}$ and $d=0.8 \mathrm{~m}$. Since there are adiabatic walls in the CFD model, the downward-facing wall flow shown in one case in Fig. 1 was not expected, though. However, the temperature difference between the thermal zones affects $v_{\text {limit }}$ massively. Hence, the behaviour of $v_{\text {limit }}$ and $\Delta T$ can roughly be approximated with a root function. 


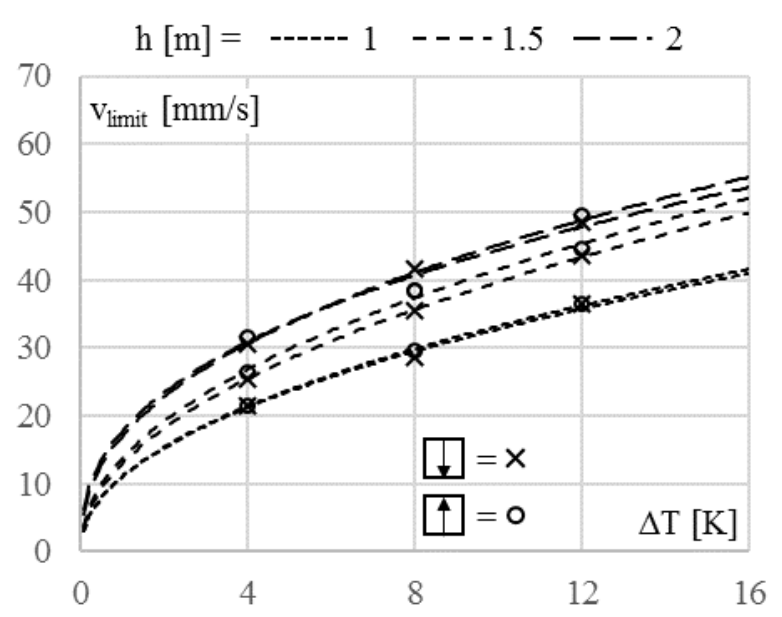

Fig. 8. $v_{\text {limit }}$ with a depth of $0.4 \mathrm{~m}$.

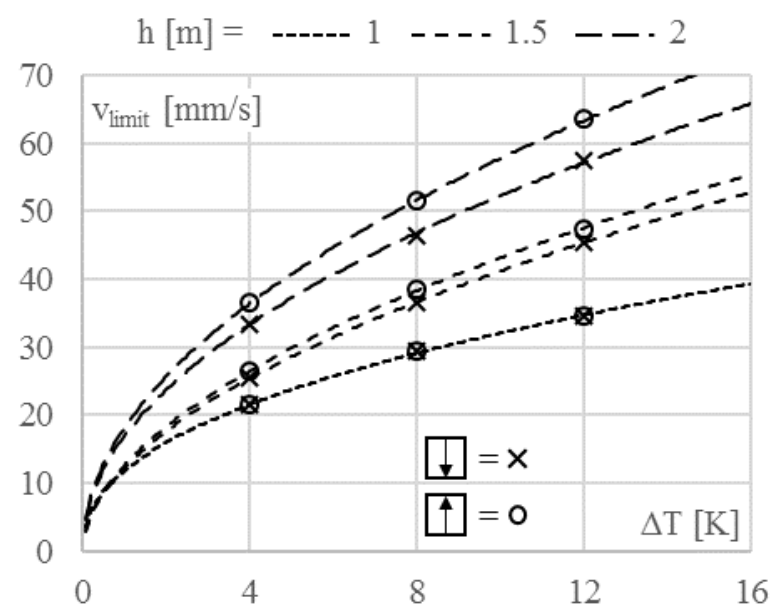

Fig. 9. $v_{\text {limit }}$ with a depth of $0.8 \mathrm{~m}$.

Furthermore, Archimedes number for $v_{\text {limit }}$ is evaluated for both values of the staircase's depth. The height of the staircase is chosen as the characteristic length.

In Fig. 10 it can be seen that the occurring Archimedes numbers are in the range of 180-320.

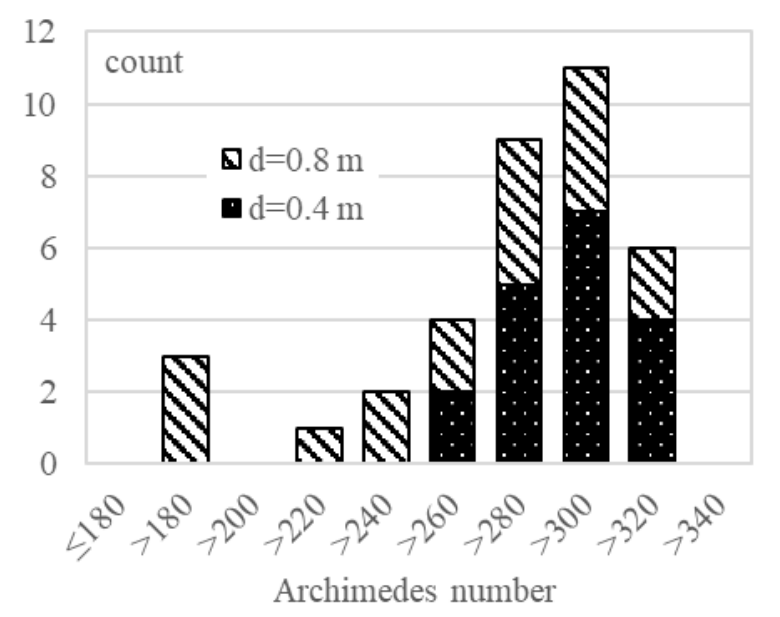

Fig. 10. Archimedes number for $v_{\text {limit }}$.
For the thinner staircase, Archimedes numbers concentrate at around 290, while for the thicker staircase there is a wide range of values. As the length ratio of the base area is around 1 for the thicker staircase, the air flow might have a higher three dimensional character than for the thinner staircase. Consequently, for the stability criteria further dimensionless numbers are necessary to fully describe the problem set.

\subsection{Analytical pressure functions}

Regarding the previous findings, temperature profiles can be approximated with analytical functions analogously to Fig. 11 in order to solve equation (4).

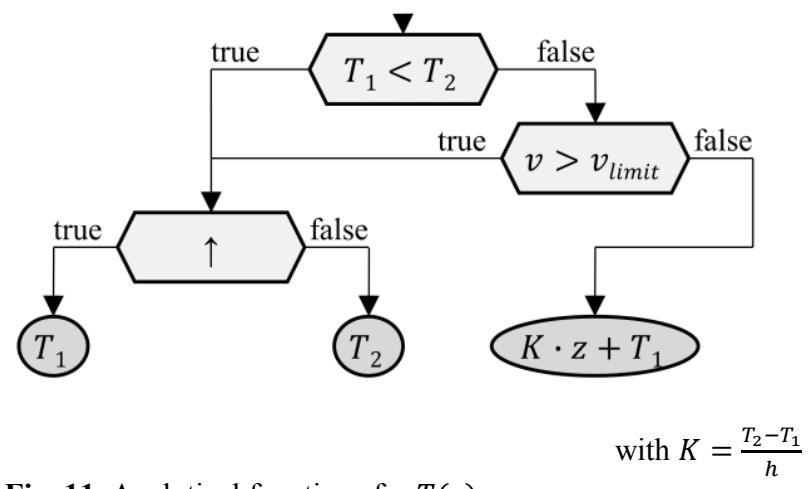

Fig. 11. Analytical functions for $T(z)$.

The case $T_{1}<T_{2}$ is trivial, because there is no thermal potential for buoyancy. Therefore, the temperature in the staircase evolves with the inflow temperature. In the other case, there is a limiting velocity, which determines the temperature characteristic as illustrated.

If the linear form is applicable, the pressure equation comes to

$$
p_{\text {lin }}=p_{0} \cdot\left(1+\frac{K \cdot Z}{T_{0}}\right)^{-\frac{g}{R \cdot K}}
$$

Reducing the gradient of the temperature profile ( $K$ goes towards zero) the temperature in the staircase becomes constant. This leads to equation (6).

$$
\lim _{K \rightarrow 0} p_{\text {lin }}=p_{\text {const }}=p_{0} \cdot e^{-\frac{g}{R \cdot T} \cdot z}
$$

Assuming the outside temperature is equal over the height of the building, equation (6) is also valid to describe the outside pressure condition.

In a holistic view, we can now assess the pressure difference at the ventilation device in order to solve equation (2).

Fig. 12 shows the case $T_{1}>T_{2}$ with buoyancy. It can be seen that if the outside temperature is equal to the mean temperature between the floors, there are balanced pressure conditions at the ventilation devices. As outlined in chapter 2.2 , for the winter case $T_{a}=0^{\circ} \mathrm{C}$ pressure differences of around $1 \mathrm{~Pa}$ occur. As solid and dashed lines collapse, the flow direction has no influence on the pressure differences. 


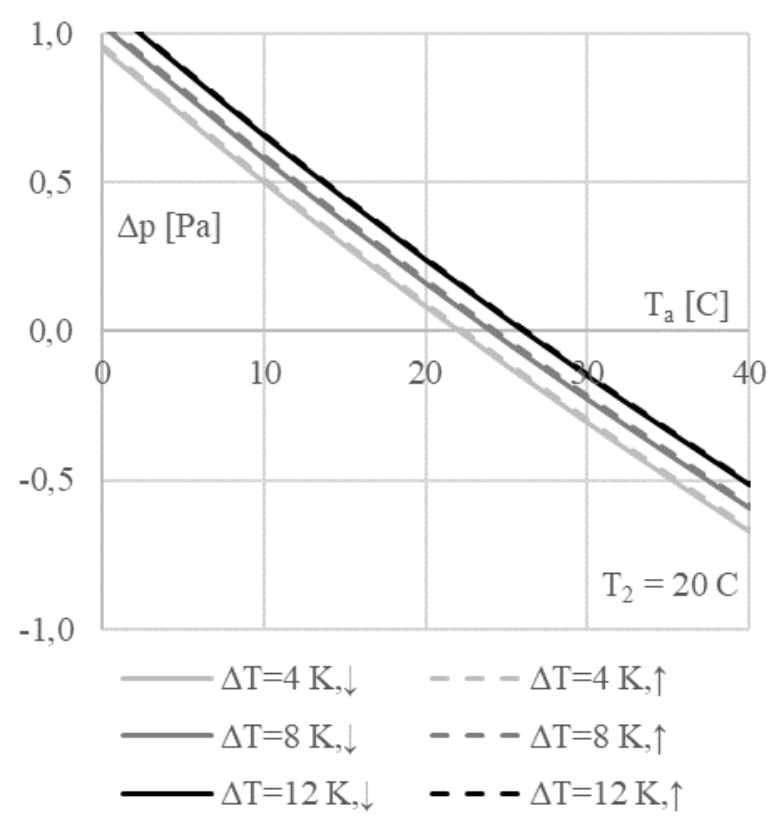

Fig. 12. Pressure difference at ventilation device $\left(T_{1}>T_{2}\right)$.

Fig. 13 represents the other case when $T_{1}<T_{2}$. Since $T_{1}=20^{\circ} \mathrm{C}$ is fixed, there are no dependencies on the temperature difference, if the ventilation air flow is directed upwards (dashed lines). Otherwise, if the flow points downwards, the expected pressure differences are higher than in Fig. 12, because the mean temperature difference is higher by the absence of buoyancy.

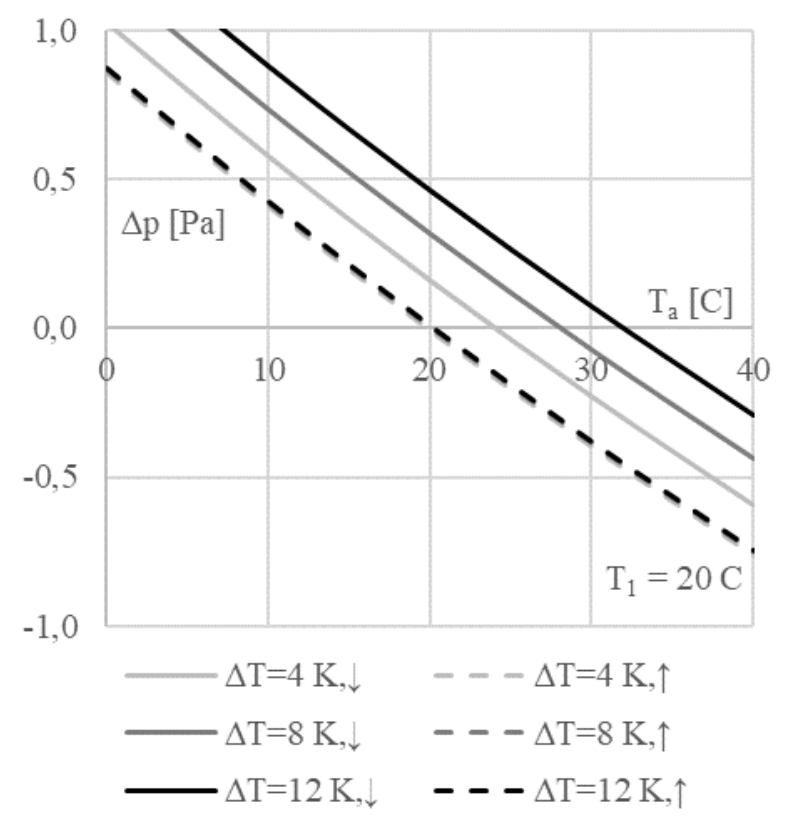

Fig. 13. Pressure difference at ventilation device $\left(T_{1}<T_{2}\right)$.

Taking the characteristic curve of the decentralized ventilation devices into account, pressure differences of 1.5 Pa can result in a deviation of the volume flow rate of around $1.5 \mathrm{~m}^{3} / \mathrm{h}$. In time of reduced ventilation power, this leads to up to $10 \%$ losses in volume flow.

\section{Discussion and Conclusion}

This paper shows that there are slight influences on the ventilation devices in a staircase case with buoyancy, but they are considerably small. Taking into consideration that there are not only two ventilation devices but several ones installed in the entire building then pressure and temperature conditions become more and more complex.

The aim of this study was to estimate the pressure conditions in a very first step and thus, several assumptions are used when comparing the staircase in reality and the model used in this paper. Nevertheless, with the CFD simulations we were able to simulate an air flow with both a vertical flow direction and buoyancy effects.

The advantages of the adjusted porous media in the CFD model have the potential to serve for further investigations with this approach. Since there are only a few parameters varied in this study, it might be possible to achieve dimensionless numbers for this problem set in the future. Apart from this, the thermal zones do not have to be placed one above but next to each other. With this setup, the flow between two differently tempered rooms can be investigated for example.

Grateful acknowledgement is made for financial support by Federal Institute for Research on Building, Urban Affairs and Spatial Development (BBSR), promotional reference SWD10.08.18.7-16.32.

\section{References}

1. A.A. Peppes, M. Santamouris, D.N. Asimakopoulos. Build Environ. 36. (2001)

2. A.A. Peppes, M. Santamouris, D.N. Asimakopoulos. Build Environ. 37. (2002)

3. R. du Puits, C. Resagk, A. Tilgner, F.H. Busse, A. J. Fluid Mech. 572. (2007)

4. W. Linke. Gesundheits-Ingenieur. (1962)

5. H. Rietschel. Lehrbuch der Heiz- und Lüftungstechnik. (1963)

6. J. Wildeboer, M. Grübbel, I. Gores, K. Fitzner. HLH. 4. (2002)

7. C. Kandzia, D. Müller. Int. Journ. of Vent. 17. (2017)

8. F.R. Menter. AIAA J. 32. (1994)

9. ANSYS, Inc. ANSYS. (2016). 